\title{
Osteoporosis Knowledge of Students in Relevant Healthcare Academic Programs
}

\author{
Vu H. Nguyen ${ }^{1}$ and Ze Wang ${ }^{2}$ \\ ${ }^{1}$ Public Health Program, Department of Health Sciences, University of Missouri, Columbia, MO 65211, USA \\ ${ }^{2}$ Department of Educational, School and Counseling Psychology, University of Missouri, Columbia, MO 65211, USA
}

Correspondence should be addressed to Vu H. Nguyen, nguyenvh@health.missouri.edu

Received 16 September 2012; Revised 20 October 2012; Accepted 28 October 2012

Academic Editor: E. M. Lewiecki

Copyright (C) 2012 V. H. Nguyen and Z. Wang. This is an open access article distributed under the Creative Commons Attribution License, which permits unrestricted use, distribution, and reproduction in any medium, provided the original work is properly cited.

\begin{abstract}
For healthcare professionals who treat individuals with osteoporosis, it is vital that they receive adequate education on osteoporosis to ensure sufficient knowledge of osteoporosis to properly treat individuals with the disease. To test for adequate osteoporosis education, a study was conducted to measure osteoporosis knowledge in 206 students in relevant healthcare academic programs, such as nursing, pharmacy, physical therapy, and dietetics. The study showed that differences existed in osteoporosis knowledge in general between the programs and between different years of students in the same programs. There were also discrepancies in specific areas of osteoporosis knowledge between the classes of students, and the average scores of correctly answered items were only as high as $24.40(76.3 \%)$ out of 32 items on osteoporosis knowledge. This study shows that students have osteoporosis knowledge and that it is not completely inadequate; however, osteoporosis knowledge could still be more sufficient, and results demonstrate the need to increase osteoporosis education in the curriculum for these healthcare academic programs to increase osteoporosis knowledge and better prepare graduates and professionals to treat individuals with the disease.
\end{abstract}

\section{Introduction}

Osteoporosis is a skeletal disease of reduced bone mineral density (BMD) that is diagnosed as 2.5 standard deviations below the adult peak mean that compromises bone strength resulting in an increased risk of bone fracture [1]. Osteoporosis affects about 10 million Americans with additional tens of millions at risk in the United States [2], and also affects hundreds of millions of people worldwide [3]. Reduced BMD due to osteoporosis leads to an increase in bone fractures that occur most frequently fractures in the hip, spine, and wrist, and these osteoporotic fractures reduce both the quality of life [4] and quantity of life [5]. In addition, treating individuals with osteoporosis costs billions of dollars per year on the nation's healthcare system [6] that is expected to continuously and greatly increase in the next couple of decades [7].

With the increased prevalence of osteoporosis, it is vital for healthcare professionals who work with individuals with osteoporosis to be adequately educated to have proficient knowledge of the disease and how to prevent and treat it. Healthcare professionals should receive adequate osteoporosis education in their academic programs in order to give them adequate knowledge of the disease to prepare them for treating this population. A systematic review of interventions by healthcare professionals on communitydwelling postmenopausal women with osteoporosis defined these interventions by consultation with physicians, nurses, pharmacists, physical therapists, and dietitians [8]. Physicians who treat individuals with osteoporosis have additional training after medical school with residencies and possible fellowships to prepare them to treat patients with the disease; therefore, this study investigated osteoporosis knowledge in students who were soon to be nurses, pharmacists, physical therapists, and dietitians.

\section{Materials and Methods}

2.1. Participants and Procedures. This study was conducted near the end of an academic year at a large university, and 
Table 1: Demographic information.

\begin{tabular}{lcccc}
\hline Class & Number of participants & Gender & Ethnicity & Age mean (years) \\
\hline Accelerated senior nursing & 38 & $86.8 \%$ Female & $94.6 \%$ Caucasian & $27.42(7.58)$ \\
Traditional senior nursing & 48 & $93.8 \%$ Female & $97.9 \%$ Caucasian & $21.81(0.57)$ \\
2nd year pharmacy & 21 & $90.5 \%$ Female & $97.9 \%$ Caucasian & $23.67(4.42)$ \\
4th year pharmacy & 16 & $87.5 \%$ Female & $93.8 \%$ Caucasian & $24.75(2.27)$ \\
1st year physical therapy & 43 & $65.1 \%$ Female & $95.3 \%$ Caucasian & $23.56(2.83)$ \\
3rd year physical therapy & 11 & $90.9 \%$ Female & $100 \%$ Caucasian & $24.45(1.37)$ \\
Junior dietetics & 14 & $100 \%$ Female & $81.8 \%$ Caucasian & $23.27(4.76)$ \\
Senior dietetics & 15 & $100 \%$ Female & $85.7 \%$ Caucasian & $22.33(0.98)$ \\
\hline
\end{tabular}

Standard deviation for age mean is in parentheses.

the university's institutional review board approved of the study. A convenience sample of 206 students in relevant healthcare academic programs participated in this study testing osteoporosis knowledge. The majority of students were female and Caucasian, with ages averaging around 21 to 27 years. Those academic programs included nursing, pharmacy, physical therapy, and dietetics. Two classes in each program were included, resulting in a total of eight classes (see Table 1).

Nursing students were seniors in both the accelerated and traditional programs graduating with a Bachelor of Science in Nursing. Pharmacy students were 4th year students who had a 5th year of Advanced Pharmacy Practice Experiences prior to graduating with a Doctor of Pharmacy. Physical therapy students were 3rd year students completing their Clinical Education and graduating with a Doctor of Physical Therapy. Dietetics students were in a Coordinated Program in Dietetics and were junior dietetics students, and senior dietetic students who had completed their Supervised Practice Experience and graduating with a Bachelor of Science with an emphasis area in Medical Dietetics. Unlike nursing and dietetics that are undergraduate programs, because pharmacy and physical therapy are professional school programs, data collected on a separate sample of 2nd year pharmacy students and 1st year physical therapy students were used to assess differences in osteoporosis knowledge for the 4 th year pharmacy students and 3rd year physical therapy students, respectively.

Each participant received a written letter of consent, and scales on demographic information and osteoporosis knowledge to voluntarily complete and submit. Data was collected anonymously, and each participant had the opportunity to sign up to enter a drawing to win one out of a set number of gift cards to a software-based, online, digital media store.

2.2. Measures. The Osteoporosis Knowledge Test (OKT) (Revised 2011) was administered. It is a revised version of the original and consists of 32 multiple-choice items by Gendler, Coviak, Martin and Kim, with an item developed and added by von Hurst [9]. The OKT has 2 subscales; the OKT Nutrition subscale has 26 items (items 1 to 11 and 18 to 32), and OKT Exercise subscale has 20 items (items 1 to 17 and 30 to 32). The total scale and 2 subscales have good internal consistency (Kuder-Richardson 20 equal 0.85 for the total scale, 0.83 for the Nutrition subscale and 0.81 for the Exercise subscale). Test-retest reliability is 0.87 . The OKT has good content validity. And it is worth noting that both the Exercise and Nutrition subscales include items 1 to 11.

Items 1 to 11 of the OKT address osteoporosis risk factors and each has 4 response options: "MORE LIKELY," "LESS LIKELY," "NEUTRAL," and "DON’T KNOW." Since the 11 items measure knowledge of osteoporosis risk factors, a subscale (Risk Factor) based on them was calculated in addition to the Exercise and Nutrition subscales. Items 12 to 32 of the OKT are all 4-item multiple-choice items with 3 different responses with a fourth response option of "Don't know." Each of the 32 items has one correct/best answer worth 1 point when scored.

2.3. Statistical Analysis. Descriptive statistics for the total OKT scale and subscales of Exercise, Nutrition, and Risk Factor knowledge were reported for the eight groups/classes of students in the four academic programs. One-way analysis of variance tests were conducted to find statistically significant differences between the eight groups.

\section{Results and Discussion}

Table 2 shows the scores on the total OKT scale and the three subscales by group. On the total OKT scale, 2nd year pharmacy students correctly answered about 19.48 items out of the 32 items, which was significantly lower than all other students except 1st year physical therapy students. Senior dietetics students correctly answered 24.40 items on the total OKT scale, the highest performing group of the eight classes. Comparing the two classes within the same program, the two classes in pharmacy were statistically significantly different, as were the two classes in physical therapy. Fourth-year pharmacy students scored about 3.8 points higher on the total OKT than the 2nd year pharmacy students. Third-year physical therapy students scored about 3.5 points higher on the total OKT than 1st year physical therapy students.

On the OKT Exercise subscale, 2nd year pharmacy students had the lowest score (11.90 out of 20), followed by 1 st year physical therapy students (13.12 out of 20). Comparing the two classes within the same program, only the two classes in pharmacy were statistically significantly different. The 4 th year pharmacy students scored 2.7 points 
TABLE 2: Total and subscale scores by class.

\begin{tabular}{lcccc}
\hline Class & Total (out of 32) & Exercise (out of 20) & Nutrition (out of 26) & Risk factor (out of 11) \\
\hline Accelerated senior nursing & $22.66(2.54)^{\mathrm{ab}}$ & $14.71(2.22)^{\mathrm{a}}$ & $18.29(1.97)^{\mathrm{a}}$ & $8.58(1.39)^{\mathrm{a}}$ \\
Traditional senior Nursing & $22.94(2.52)^{\mathrm{cd}}$ & $14.83(2.11)^{\mathrm{bc}}$ & $18.65(2.21)^{\mathrm{bc}}$ & $8.54(1.47)^{\mathrm{bc}}$ \\
2nd year pharmacy & $19.48(3.64)^{\mathrm{accefgh}}$ & $11.90(2.76)^{\mathrm{abde}}$ & $16.29(3.20)^{\mathrm{bdef}}$ & $6.90(2.02)^{\mathrm{abde}}$ \\
4th year pharmacy & $23.25(3.66)^{\mathrm{ei}}$ & $14.63(2.25)^{\mathrm{d}}$ & $18.94(3.02)^{\mathrm{dg}}$ & $8.25(2.08)^{\mathrm{d}}$ \\
1st year physical therapy & $20.33(3.61)^{\mathrm{bdijkl}}$ & $13.12(2.73)^{\mathrm{c}}$ & $16.35(2.89)^{\mathrm{acghi}}$ & $7.33(1.55)^{\mathrm{c}}$ \\
3rd year physical therapy & $23.82(2.09)^{\mathrm{fj}}$ & $15.55(1.81)^{\mathrm{e}}$ & $18.82(1.78)$ & $9.00(1.20)^{\mathrm{e}}$ \\
Junior dietetics & $23.71(3.02)^{\mathrm{gk}}$ & $13.64(3.18)$ & $19.07(2.40)^{\mathrm{eh}}$ & $7.29(1.82)$ \\
Senior dietetics & $24.40(2.35)^{\mathrm{hl}}$ & $13.73(2.15)$ & $19.87(1.96)^{\mathrm{fi}}$ & $7.40(1.77)$ \\
\hline$F(7,198)$ & 7.77 & 5.21 & 6.73 & 5.21 \\
$P$ & $<.001$ & $<.001$ & $<.001$ & $<.001$ \\
Effect size $\left(\eta_{\text {partial }}^{2}\right)$ & 0.216 & 0.156 & 0.192 & 0.156 \\
\hline
\end{tabular}

Standard deviations are in parentheses. Values in the same column with the same superscript are statistically different at .05 level after Bonferroni adjustment.

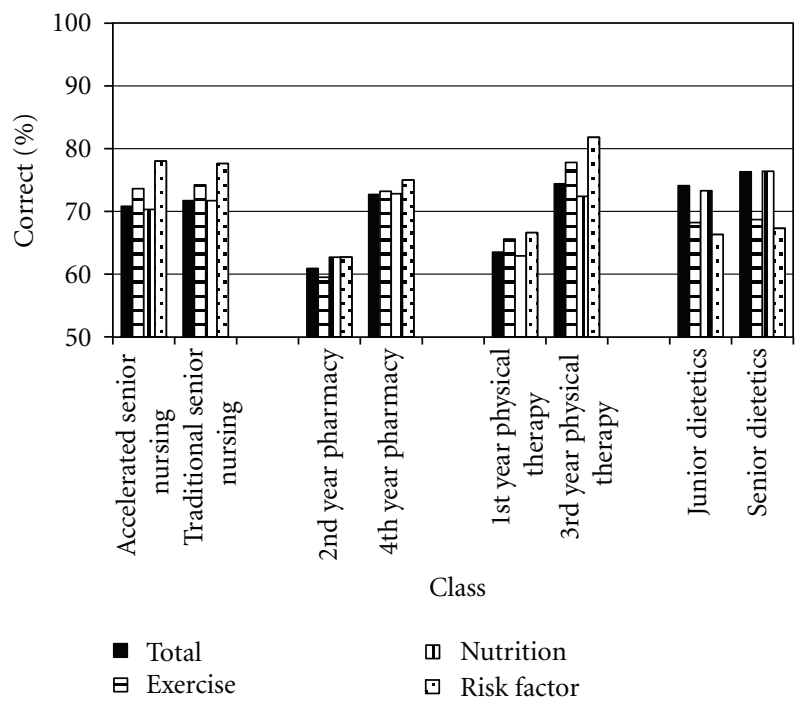

Figure 1: Percent correct for total and subscales by class.

higher than 2nd year pharmacy students. On the OKT Nutrition subscale, 2nd year pharmacy students had the lowest score (16.29 out of 26), followed by 1st year physical therapy students (16.35 out of 26). Comparing the two classes within the same program, only the two classes in pharmacy were significantly different. Fourth-year pharmacy students scored 2.7 points higher than 2nd year pharmacy students. On the Risk Factor subscale, 2nd year pharmacy students had the lowest score (6.90 out of 11), followed by junior dietetics students (7.29 out of 11). Comparing the two classes within the same program, only the two classes in pharmacy were significantly different, as fourthyear pharmacy students scored 1.4 points higher than 2nd year pharmacy students. When comparing the two classes within the same program, education in pharmacy and physical therapy school showed to significantly increase total osteoporosis knowledge.

Figure 1 illustrates the percentage of correctly answered items for the total OKT and the Exercise, Nutrition, and Risk
Factor subscales. For the total OKT, senior dietetics students scored the highest at $76.3 \%$, and all other classes scored over $70 \%$ correctly with the exception of 2nd year pharmacy $(60.9 \%)$ and 1 st year physical therapy (63.5\%). Comparing the Exercise and Nutrition subscales, both nursing classes and both physical therapy classes scored higher on the Exercise subscales than Nutrition subscales, and both dietetics classes scored higher on the Nutrition subscale than Exercise subscale. With the exception of the dietetics students, all other classes scored the highest percent correctly for Risk Factor knowledge than for the total OKT and Exercise and Nutrition subscales.

With the exception of the 2nd year pharmacy and 1st year physical therapy students, all other classes scored over $70 \%$ on the total OKT, ranging from $70.8 \%$ for the accelerated nursing students to $76.3 \%$ for the senior dietetics students. Using a typical university scoring scale of $A=90$ to $100 \%$, $B=80$ to $89.9 \%, C=70$ to $79.9 \%, D=60$ to $69.9 \%$, and $F=$ less than $60 \%$, the average total scores on the OKT would merit a grade from a $C-$ to $C$. This shows that total osteoporosis knowledge is not completely inadequate, but still not enough to display sufficient knowledge to treat individuals with the disease as scores between 80 and $89.9 \%$ that merit a $B$ grade or between 90 and $100 \%$ that merit an $A$ grade were not obtained by any class.

Physical therapy students study physical activity extensively with minimal nutrition education in their academic program, and not surprisingly, scored higher on the Exercise subscale than on the Nutrition subscale, and also notably scored highest on the Risk Factor subscale. Third-year physical therapy students were the only class to score over $80 \%$ on any scale with a score of $81.8 \%$ on the Risk Factor subscale. Nursing students also scored higher on the Exercise subscale than on the Nutrition subscale. Dietetics students study diet extensively with minimal physical activity education in their academic program, and not surprisingly, scored higher on the Nutrition subscale than on the Exercise subscale; however, notably scored lowest on Risk Factor subscale out of all scales. Junior and senior dietetic students unsurprisingly scored the highest of all classes in the Nutrition subscale with scores of $73.3 \%$ and $76.4 \%$, respectively, but scored less than 
$70 \%$ on both the Exercise and Risk Factor subscales. The significant difference in the total score between 1st year and 3rd year physical therapy students show general osteoporosis education in their curriculum. The significant difference in the total and three subscales between 2nd year and 4th year pharmacy students show more overall osteoporosis education in the curriculum covering osteoporosis, exercise and nutrition to prevent and treat osteoporosis, and risk factors of osteoporosis. However, in consideration of all of the scores, in total osteoporosis knowledge and each individual subscale of exercise, nutrition, and risk factors, it is apparent that all classes could increase overall osteoporosis education for improved scores in all areas.

This study shows consistent results from previous studies. Osteoporosis knowledge has been previously studied in students and practitioners in nursing. For nursing students, Berarducci [10] found that senior nursing students had limited knowledge of osteoporosis magnitude, risk factors, detection, treatment, and preventive measures, and suggested that this evidence should be used to enhance the curriculum covering osteoporosis. But nursing students do receive some osteoporosis education in their curriculum, as Ziccardi et al. [11] found that senior nursing students had higher osteoporosis knowledge than sophomore nursing students. However, the results of those previous studies and this current study show that there is a need to increase osteoporosis education in the nursing curriculum, as osteoporosis knowledge could still be more sufficient to treat individuals with the disease. And to the knowledge of the researchers, osteoporosis knowledge has not been investigated in pharmacy, physical therapy, or dietetics students to determine whether or not they had received adequate education on osteoporosis in their respective curriculums, at least not with the OKT. Nevertheless, results from this study show that osteoporosis knowledge could still be more sufficient in students in each academic program for better preparation to treat individuals with osteoporosis.

This study was not without limitations. Convenience sampling was of a population on the campus at one university. Although these healthcare academic curriculums have met the same standards of other accredited healthcare academic programs throughout the nation, having the entire sample taken on the campus at one institution may not represent osteoporosis knowledge for students studying these particular healthcare fields in other locations. Random responding due to low motivation and/or capability was also of concern. Although this study assessed osteoporosis knowledge in each healthcare academic program, the type and amount of osteoporosis content in each healthcare academic program's curriculum was not examined.

\section{Conclusions}

From the results of this study, we conclude that osteoporosis knowledge is not completely inadequate and students do have knowledge of osteoporosis. However, we feel that osteoporosis knowledge is still not sufficient for students in each of these disciplines, and that there is a need for increased osteoporosis education in all of their healthcare academic curriculums, with emphasis on exercise and nutrition to prevent and treat osteoporosis, and on osteoporosis risk factors. With an increase in osteoporosis education for students studying nursing, pharmacy, physical therapy, and dietetics, graduates of these healthcare academic programs should have higher osteoporosis knowledge and would be better prepared to treat individuals with osteoporosis.

\section{References}

[1] J. A. Kanis, L. J. Melton, C. Christiansen, C. C. Johnston, and N. Khaltaev, "Perspective: the diagnosis of osteoporosis," Journal of Bone and Mineral Research, vol. 9, no. 8, pp. 11371141, 1994.

[2] U.S. Department of Health and Human Services, Bone Health and Osteoporosis: A Report of the Surgeon General, U.S. Department of Health and Human Services: Office of the Surgeon General, Rockville, Md, USA, 2004.

[3] C. Cooper, G. Campion, and L. J. Melton, "Hip fractures in the elderly: a world-wide projection," Osteoporosis International, vol. 2, no. 6, pp. 285-289, 1992.

[4] P. Lips and N. M. van Schoor, "Quality of life in patients with osteoporosis," Osteoporosis International, vol. 16, no. 5, pp. 447-455, 2005.

[5] O. Johnell, J. A. Kanis, A. Odén et al., "Mortality after osteoporotic fractures," Osteoporosis International, vol. 15, no. 1, pp. 38-42, 2004.

[6] N. F. Ray, J. K. Chan, M. Thamer, and L. J. Melton, "Medical expenditures for the treatment of osteoporotic fractures in the United States in 1995: report from the National Osteoporosis Foundation," Journal of Bone and Mineral Research, vol. 12, no. 1, pp. 24-35, 1997.

[7] R. Burge, B. Dawson-Hughes, D. H. Solomon, J. B. Wong, A. King, and A. Tosteson, "Incidence and economic burden of osteoporosis-related fractures in the United States, 20052025," Journal of Bone and Mineral Research, vol. 22, no. 3, pp. 465-475, 2007.

[8] P. Lai, S. S. Chua, and S. P. Chan, "A systematic review of interventions by healthcare professionals on communitydwelling postmenopausal women with osteoporosis," Osteoporosis International, vol. 21, no. 10, pp. 1637-1656, 2010.

[9] P. Gendler, C. Coviak, J. Martin, K. Kim, and P. von Hurst, Osteoporosis Knowledge Test (Revised 2011), Grand Valley State University, Allendale, Mich, USA, 2011.

[10] A. Berarducci, "Senior nursing students' knowledge of osteoporosis," Orthopaedic Nursing, vol. 23, no. 2, pp. 121-127, 2004.

[11] S. L. Ziccardi, C. A. Sedlak, and M. O. Doheny, "Knowledge and health beliefs of osteoporosis in college nursing students," Orthopaedic Nursing, vol. 23, no. 2, pp. 128-133, 2004. 


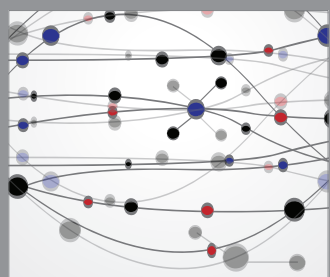

The Scientific World Journal
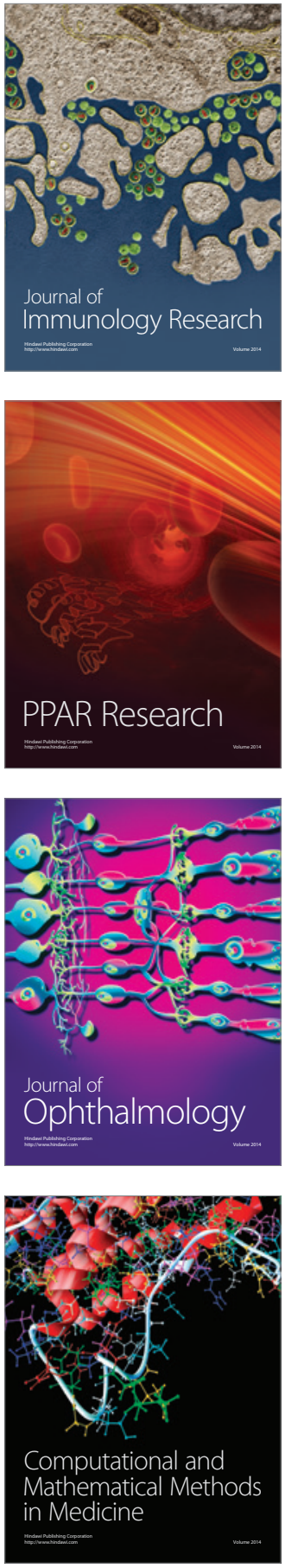

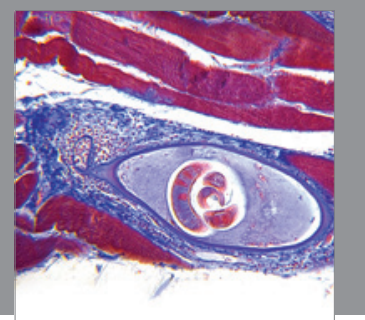

Gastroenterology

Research and Practice
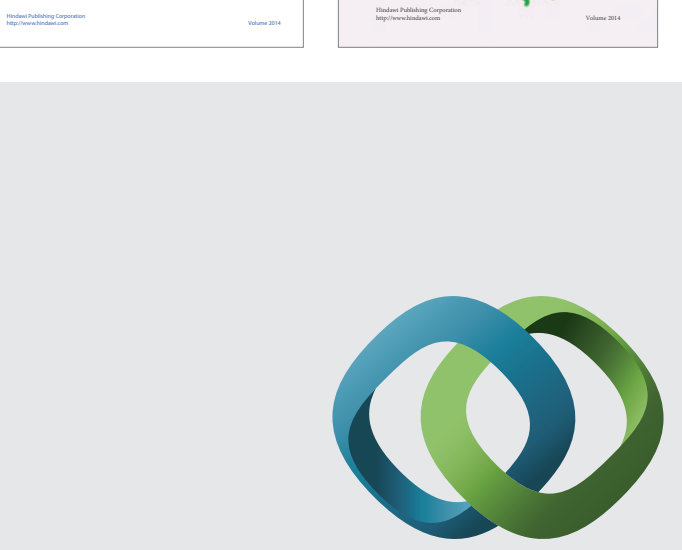

\section{Hindawi}

Submit your manuscripts at

http://www.hindawi.com
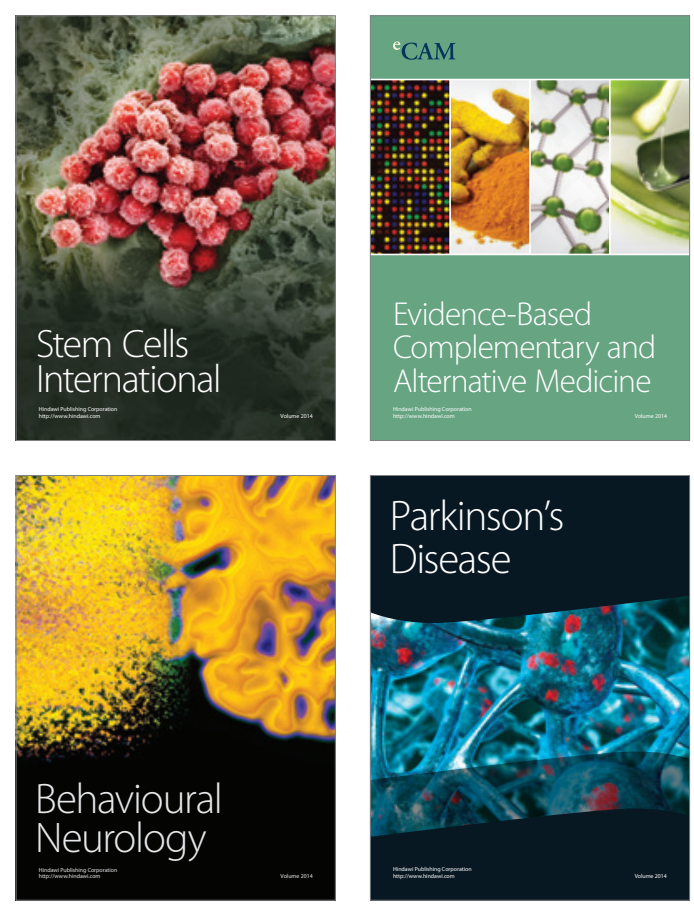

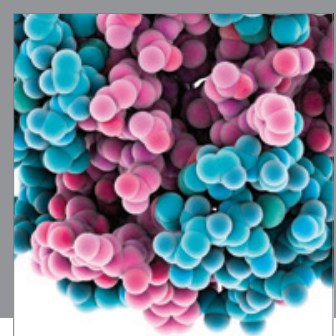

Journal of
Diabetes Research

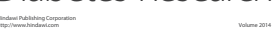

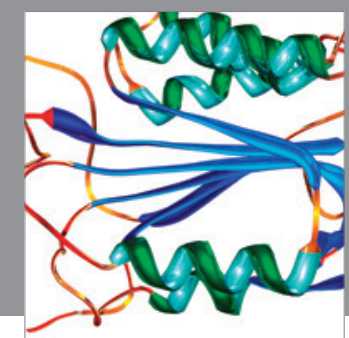

Disease Markers
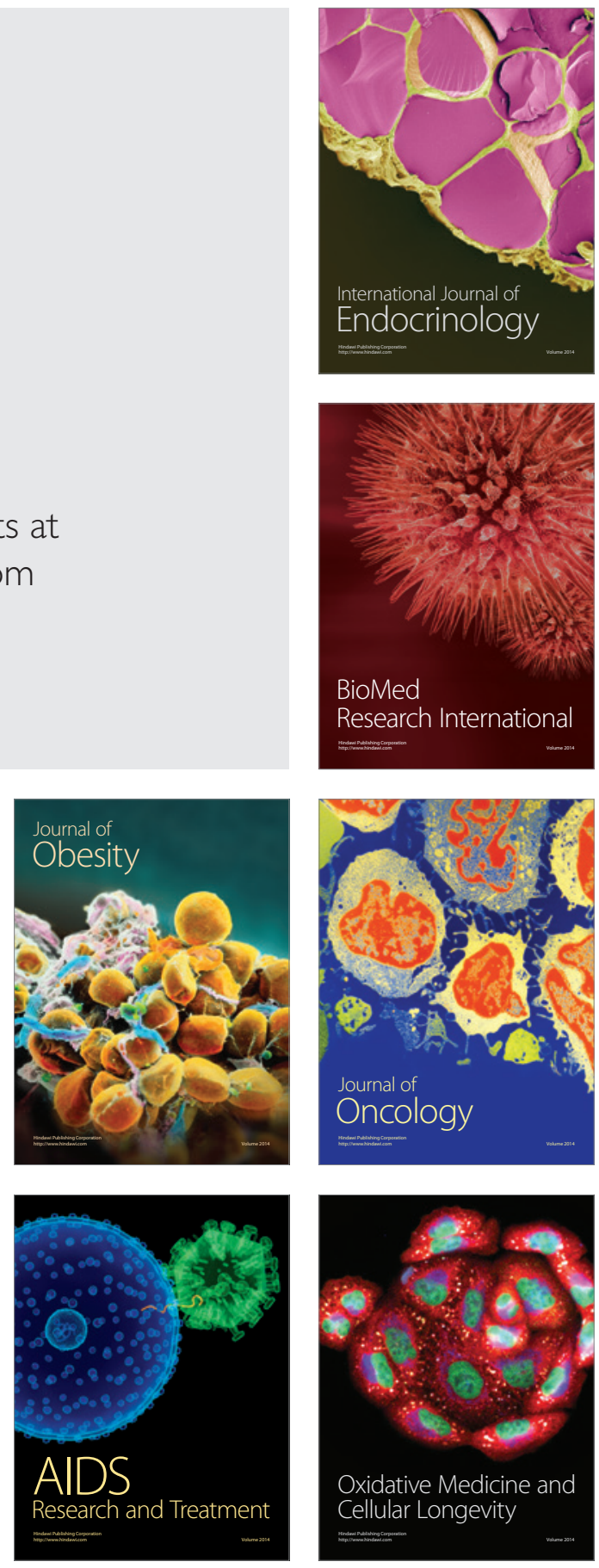